\title{
Inteligencia emocional y burnout en docentes de educación inicial de
}

\section{Ayacucho}

\section{Emotional intelligence and burnout in early education teachers in Ayacucho}

\author{
Camila Abarca Gálvez ${ }^{1}$, Lucía Ramírez Gutiérrez ${ }^{2}$ y Tomás Caycho-Rodríguez ${ }^{3 a}$ \\ Universidad Peruana de Ciencias Aplicadas, Lima, Perú ${ }^{12}$ \\ Universidad Privada del Norte, Lima, Perú ${ }^{3}$ \\ (iD) Orcid ID: https://orcid.org/0000-0002-3197-6167 1 \\ (iD) Orcid ID: https://orcid.org/0000-0002-4549-2075² \\ (iD) Orcid ID: https://orcid.org/0000-0002-5349-7570 3
}

Recibido: 10 de setiembre 2019

Aceptado: 30 de enero 2020

\section{Resumen}

El presente estudio relaciona la inteligencia emocional y el burnout en una muestra de 294 docentes de genero femenino de educación inicial en Ayacucho, de 23 a 65 años $\left(M_{\text {edad }}=40\right)$. Se utilizó la Escala de Inteligencia Emocional (WLEIS) y el Cuestionario de Burnout del Profesorado Revisado (CBP-R). Los resultados reportaron que existen correlaciones negativas entre el burnout y el uso $\left(\mathrm{r}_{\text {núcleo del burnout }}=-.38 ; \mathrm{r}_{\text {falta de realización }}=-.33 ; \mathrm{p}<.01\right)$, regulación $\left(\mathrm{r}_{\text {núcleo del burnout }}=-.28 ; \mathrm{r}_{\text {falta de realización }}\right.$ $=-.26 ; \mathrm{p}<.01$ ), valoración de las propias emociones ( $\mathrm{r}_{\text {burnout nucleus }}=-.25 \mathrm{r}_{\text {falta de realización }}=-.21 ; \mathrm{p}<$ .01). Por otro parte, al comparar la inteligencia emocional y el burnout con la situación laboral, se determinó que las docentes nombradas se sienten realizadas $(\mathrm{U}=8328, z=-2.35, p=.02, \mathrm{RP}=$ $.42)$, usan sus propias emociones $(\mathrm{U}=8417, z=-2.23, p=.03, \mathrm{RP}=.42)$ y valoran sus emociones $(\mathrm{U}=8330.50, z=-2.35, p=.02, \mathrm{RP}=.42)$, a diferencia de las contratadas. En relación con los estudios de segunda especialidad y diplomados, las docentes que poseen dicha formación usan sus propias emociones $(\mathrm{U}=8615, z=-2.33, p=.02, \mathrm{RP}=.42)$, a diferencia de aquellas que no cuentan con dichos estudios. Finalmente, las maestras con auxiliar en el aula usan las propias emociones $(\mathrm{U}=7942, z=-3.33, p=.00, \mathrm{RP}=.39)$, valoran sus emociones $(\mathrm{U}=8870.5, z=-2.01, p=.04$, $\mathrm{RP}=.43)$ y se sienten realizadas $(\mathrm{U}=8686, z=-2.27, p=.02, \mathrm{RP}=.42)$ a diferencia de aquellas que no cuentan con auxiliar.

Palabras clave: Inteligencia emocional, Burnout, educación inicial, Ayacucho.

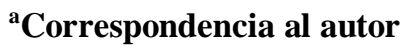

E-mail: tomas.caycho@upn.pe
} 


\begin{abstract}
This research relates emotional intelligence and burnout in a sample of 294 female teachers of initial education in Ayacucho, aged 23 to 65 (Medad =40). The Emotional Intelligence Scale (WLEIS) and the Revised Teacher Burnout Questionnaire (CBP-R) were used. The results reported negative correlations between burnout and use $\left(\mathrm{r}_{\text {burnout nucleus }}=-.38 ; \mathrm{r}_{\text {lack of realization }}=-.33\right.$; $\mathrm{p}<.01$ ), regulation ( $\mathrm{r}_{\text {burnout nucleus }}=-.28 ; \mathrm{r}_{\text {lack of realization }}=-.26 ; \mathrm{p}<.01$ ), valuation of one's own emotions ( $\mathrm{r}_{\text {burnout nucleus }}=-.25 \mathrm{r}_{\text {lack of realization }}=-.21 ; \mathrm{p}<.01$ ). On the other hand, when comparing the emotional intelligence and the burnout with the employment situation, it was determined that the appointed teachers feel fulfilled $(\mathrm{U}=8328, \mathrm{z}=-2.35, \mathrm{p}=.02, \mathrm{RP}=.42)$, use their own emotions $(\mathrm{U}=8417, \mathrm{z}=-2.23, \mathrm{p}=.03, \mathrm{RP}=.42)$, and value their emotions $(\mathrm{U}=8330.50, \mathrm{z}=-$ $2.35, \mathrm{p}=.02, \mathrm{RP}=.42)$, unlike the hired ones. In relation to second-degree and graduate studies, masters who have such training use their own emotions $(\mathrm{U}=8615, z=-2.33, p=.02, \mathrm{RP}=.42)$, unlike those who do not have such studies. Finally, teachers with classroom assistant use their own emotions $(\mathrm{U}=7942, z=-3.33, p=.00, \mathrm{RP}=.39)$, and value their emotions $(\mathrm{U}=8870.5, z=$ $-2.01, p=.04, \mathrm{RP}=.43)$ and feel fulfilled $(\mathrm{U}=8686, z=-2.27, p=.02, \mathrm{RP}=.42)$ contrary to those who do not have that support.
\end{abstract}

Keywords: Emotional Intelligence, Burnout, Ayacucho, Pre-school education.

\title{
Introducción
}

La educación básica en el Perú presenta limitaciones que interfieren en el adecuado desenvolvimiento de los docentes y se relacionan con el tipo de contrato de trabajo, la presencia o ausencia de materiales educativos, la metodología de enseñanza, la infraestructura de las instituciones, las exigencias del Ministerio de Educación (MINEDU), la excesiva cantidad de alumnos por aula y las dificultades entre compañeros, directores, padres de familia y comunidad escolar (De Vera García \& Gabari, 2019; Guadalupe, et al., 2017). Estos problemas poseen sus propias variaciones de acuerdo con el nivel de educación que se imparte (inicial, primario y secundario).

En este sentido, el trabajo docente en educación inicial requiere de habilidades específicas para brindar una formación integral a niños menores de seis años. La labor de los docentes de educación inicial va dirigida a lograr en el(la) niño(a) un adecuado desarrollo biológico, psicológico y social, además de prepararlos para el aprendizaje de habilidades cognitivas, motoras y sociales en el futuro y motivarlos para no desertar de la escuela (Minedu, 2016). Es así que, distintos países han mostrado su interés por priorizar el sistema educativo desde el nivel preescolar y promover la enseñanza de calidad (Markova, 2017).

En el Perú, cada región del país presenta sus propias particularidades en referencia a la 
educación. Específicamente, en el departamento de Ayacucho existen 2018 docentes y 31,046 alumnos en el nivel de educación inicial (MINEDU, 2017). Asimismo, se ha presentado un aumento de los recursos financieros por alumno. Desde del año 2013, el crecimiento anual de gasto por alumno aumentó en un $17.1 \%$ para la educación inicial, lo que indica una inversión anual de 1550 dólares por estudiante (MINEDU, 2015). Esto ha generado un aumento del 80.4\% en las matrículas a instituciones educativas del estado; asimismo, el capital destinado al área de infraestructura creció en un 33.8\% en el 2014. Sin embargo, esto solo ha beneficiado al $40.5 \%$ de las escuelas (MINEDU, 2015). El aumento de la cantidad de estudiantes y la falta de una estructura completa y adecuada para llevar a cabo las labores de enseñanza y aprendizaje generan nuevos retos para los docentes de educación inicial al momento de realizar su labor educativa. En este aspecto, una jornada laboral exhaustiva puede generar una pérdida de ánimo, disminución de la creatividad, baja motivación, menor optimismo, así como sentimientos de hostilidad hacia los niños y padres de familia (Almeida et al., 2015), lo que podría estar evidenciando algunos síntomas del burnout.

Cabe señalar que existen diversos estudios (internacionales y nacionales) sobre los índices de prevalencia de cada dimensión del burnout en docentes. En México, una muestra de 300 docentes de educación primaria permitió observar la existencia del burnout, en lo cual el $25.9 \%$ presenta altos niveles en la dimensión de agotamiento emocional, el $21.6 \%$ baja realización personal y el 80\% despersonalización. Asimismo, en Colombia, una muestra de 240 maestros evidenció que el 37\% posee un elevado agotamiento emocional y el 34\% presenta elevada despersonalización. Mientras que, en Perú, se ha registrado en un estudio de 264 docentes de primaria, que el $43.2 \%$ presenta altos niveles de burnout; el $33.7 \%$ evidencia niveles elevados de la dimensión agotamiento emocional, 33\% en despersonalización y 50\% en baja realización personal (Arias, Huamani, \& Ceballos, 2019).

En tal sentido, Maslach y Jackson (1981), García, Escorcia y Pérez (2017), y Posada, et al. (2019) mencionan que el burnout es una respuesta al estrés laboral crónico que conlleva a la vivencia de encontrarse emocionalmente agotado, el desarrollo de actividades y sentimientos negativos hacia las personas con las que se trabaja y la aparición de los procesos de devaluación del propio rol profesional; a su vez, detallan que este síndrome está constituido por tres dimensiones: el agotamiento emocional, la despersonalización y la falta de realización. Del mismo modo, el burnout puede generar consecuencias a nivel físico, social y psicológico, como fatiga 
crónica, dolores de cabeza, pecho, actitudes negativas hacia los demás, distanciamiento de su entorno, ideas de fracaso, pérdida de expectativas, entre otros (García et al., 2017).

La presencia del burnout se puede prevenir en base a diversos aspectos sociales, emocionales o cognitivos del maestro al momento de su desempeño, donde uno de los más importantes es la inteligencia emocional (Serrano, Pocinho, \& Aragón, 2018). Cabe señalar que Aguado (2016), menciona que las docentes necesitan un manejo adecuado de sus propias emociones y sentimientos para desarrollar una óptima labor; lo que les permite manejar el estrés laboral y evitar la aparición del burnout. Es así que, Herrero (2017) detalla que otro de los aspectos que beneficia a los profesionales es el desarrollo de sus habilidades sociales para potenciar sus labores cotidianas.

En relación con lo mencionado anteriormente, los docentes requieren un adecuado desarrollo de habilidades psicológicas que les permita manejar su labor. En este sentido, la inteligencia emocional posee una función preventiva en las docentes al brindar estrategias de afrontamiento para manejar el estrés laboral (Serrano et al., 2018). Tradicionalmente, la inteligencia emocional se define como la capacidad de percibir, valorar y expresar emociones con exactitud, conformada por habilidades únicas que implican el procesamiento de información afectiva y que a su vez integra rasgos de personalidad para poder desenvolverse en cualquier entorno de manera adecuada (Barcelar \& Martín, 2019; Davies, Stankouv, \& Roberts, 1998; Mayer \& Salovey, 1997; Merino, et al., 2016; Usán \& Salavera, 2019). Un docente emocionalmente inteligente es capaz de identificar, comprender y regular las emociones (Ilaja \& Reyes, 2016). Además, aquellos que posean altos índices de inteligencia emocional podrán afrontar los inconvenientes que se susciten en sus tareas diarias (Torres \& Cobo, 2016; Suárez \& Martín, 2019).

En este contexto, el objetivo del estudio es relacionar la inteligencia emocional y el burnout en docentes de educación inicial estatal de Ayacucho. Específicamente, se busca comparar la inteligencia emocional y el burnout en función a la edad, tiempo de servicio, situación laboral, estudios de segunda especialidad y diplomados; y apoyo de un auxiliar en el aula.

\section{Método}

\section{Participantes}

En el presente estudio transversal con diseño correlacional, la selección de los participantes se realizó a través de un muestreo no probabilístico por conveniencia (Otzen \& Manterola, 2017). Los criterios de inclusión fueron: 1) docentes de sexo femenino, 2) que laboren en el nivel inicial y 3) cuyo centro de trabajo esté ubicado en el departamento de Ayacucho. Para la determinación 
del tamaño de la muestra se utilizó el programa G*Power versión 3.1.9.2 (Faul et al., 2007). Para el cálculo, se utilizó un contraste de hipótesis bilateral, con un tamaño de efecto de 0.30 , un nivel de significancia de .05 y un nivel de potencia de .95 . El resultado de este análisis arrojó una cantidad mínima de 125 docentes a evaluar. En la investigación participaron 294 docentes mujeres de educación inicial estatal del departamento de Ayacucho con edades entre 23 a 65 años $(M=40$, $D E=9.25)$, con un tiempo de servicio que oscila entre 1 y 42 años $(M=12, D E=9.12)$. El 21.4\% de docentes posee segunda especialidad, el 20.4\% diplomado, el 19\% maestría, el 0.3\% ha realizado un doctorado y el $60.9 \%$ cuenta con apoyo de un auxiliar en el aula. El $36.1 \%$ son nombradas.

\section{Instrumentos}

Ficha sociodemográfica: contiene nueve ítems que recogen información acerca de la edad, tiempo de servicio, estudios de segunda especialidad y diplomados, situación laboral (nombrado y contratado), y si cuenta con auxiliar en el aula.

Inteligencia Emocional: Se utilizó la Escala de Inteligencia Emocional (WLEIS) en adultos (Wong \& Law, 2002) y validada en el Perú por Merino et al. (2016). Está compuesta por 16 ítems con un formato de respuesta tipo Likert de 7 opciones de respuesta $(1=$ completamente en desacuerdo, hasta 7 = completamente de acuerdo) que se agrupan en 4 factores: valoración y expresión de las emociones propias, valoración y reconocimiento de las emociones en otros, regulación de las propias emociones y el uso de la emoción para facilitar el desempeño. En este estudio se realizó un análisis factorial de la prueba que sugirió una estructura coherente con el modelo original que explica el 56.30\% de la varianza total. Asimismo, la consistencia interna de la dimensión uso de las propias emociones obtuvo un coeficiente alfa de cronbach adecuado de $\alpha=.79$ (IC 95\% = .74, .83). La dimensión regulación de emociones posee un coeficiente de $\alpha=.88$ $($ IC $95 \%=.85, .90)$. La dimensión valoración de propias emociones tuvo un coeficiente adecuado de $\alpha=.80$ (IC 95\% = .75, .84). Por último, en la dimensión valoración de las emociones de otros se encontró un coeficiente adecuado de $\alpha=.74$ (IC 95\% = 68, .79).

Burnout: Se empleó el cuestionario de Burnout del Profesorado Revisado (CBP-R; MorenoJiménez, Garrosa, \& González, 2000) que cuenta con una validación peruana (Moreno-Jiménez et al., 2010). El instrumento original completo posee 66 ítems; no obstante, para este estudio, se utilizó únicamente el factor burnout, conformada por 19 ítems, con cinco opciones de respuesta (1 $=$ totalmente en desacuerdo, hasta $5=$ totalmente de acuerdo) que se agrupan en tres dimensiones: 
agotamiento emocional, despersonalización y falta de realización. El análisis de la estructura interna de la prueba sugirió la presencia de dos factores: uno denominado "núcleo del burnout," que agrupó las dimensiones agotamiento emocional y despersonalización, mientras que el otro se denominó "falta de realización.” El ítem 18 (“Si un buen amigo/a me dijera que estaba interesado en tener un trabajo aquí, tendría serias reservas en recomendárselo") presentó una carga factorial menor a .30, motivo por el cual se decidió suprimirla. Finalmente, el coeficiente alfa de cronbach de la dimensión núcleo del burnout fue $\alpha=.88$ (IC 95\% $=.85, .90$ ) y para la dimensión falta de realización fue $\alpha=.75$ (IC 95\% $=.70, .80)$.

\section{Procedimiento}

Se solicitó la aprobación de los autores de cada instrumento utilizado para su aplicación en la muestra. Posterior a ello, se gestionó la autorización de la Unidad de Gestión Educativa Local (UGEL) de Huamanga para la aplicación de los instrumentos en las instituciones educativas. Asimismo, se procedió también a informar a las docentes sobre la naturaleza del trabajo y se les solicitó su colaboración como participantes, firmando cada una de ellas el consentimiento respectivo. Las docentes que aceptaron participar recibieron un cuadernillo con la ficha sociodemográfica, la escala WLEIS y el CBP-R. La aplicación se realizó entre los meses de setiembre y noviembre del 2018. No se eliminaron cuadernillos dado que las investigadoras verificaron que todos los datos estén completos. La evaluación se desarrolló de manera individual y/o grupal de acuerdo a la disponibilidad de cada maestra en las diversas instituciones a las que se acudió. El tiempo aproximado de ejecución fue de veinte minutos por persona.

\section{Análisis de Datos}

Una vez recolectados los datos, se trasladó la información obtenida al programa Statistical Package for the Social Sciences versión 24 (SPSS v. 24) y se ejecutó el análisis requerido para lograr los objetivos propuestos en la investigación. Inicialmente, se realizó el análisis psicométrico de los instrumentos (CBP-R y WLEIS), el cual estuvo constituido, en primer lugar, por el análisis descriptivo de los ítems de cada instrumento. Luego, se procedió con el análisis de homogeneidad del ítem que engloba la correlación ítem-test corregida $\left(r_{i t c}\right)$. Posterior a ello, se evaluó las evidencias de validez basadas en la estructura interna mediante el análisis factorial exploratorio (AFE) a través del método de factorización de ejes principales y la rotación oblimin. Asimismo, se estimó la confiabilidad por el método de consistencia interna a partir del cálculo del coeficiente alfa de Cronbach. Por otro lado, se examinó la distribución normal en base a la prueba de 
Kolmogorov-Smirnov. Se utilizó la prueba de correlación de Spearman y la prueba no paramétrica de U de Mann Whitney, a fin de comparar la inteligencia emocional y el burnout en función de las variables sociodemográficas. Finalmente, se estimó el tamaño del efecto (TE) a partir del cálculo de la probabilidad de superioridad (PS), donde valores de $0.56,0.64$ y 0.71 indican un TE pequeño, moderado y grande respectivamente (Grissom, 1994; Ventura-León, 2017).

\section{Resultados}

A fin de realizar el análisis de normalidad de datos en la distribución de los puntajes que existen en las dimensiones de ambas variables se ha utilizado la prueba de Kolmogorov-Smirnov. En dicha prueba se evidencia que los p-valor de todas las dimensiones son menores a .05; lo cual refleja la ausencia de normalidad en la distribución de los puntajes.

La tabla 1 indica que existe una correlación negativa, significativa y de magnitud moderada entre las dimensiones: uso de las propias emociones, núcleo del burnout y falta de realización, donde un menor núcleo de burnout está asociado con un mayor uso de las propias emociones. Del mismo modo, a menor falta de realización, mayor uso de las propias emociones. Asimismo, se observa una correlación negativa, significativa y de baja magnitud en la relación de las dimensiones regulación de emociones, núcleo del burnout y falta de realización. Es decir, a menor núcleo del burnout, mayor regulación de emociones; mientras que, una menor falta de realización está asociada con una mayor regulación de emociones. Igualmente, se manifiesta una correlación negativa, significativa y de baja magnitud entre las dimensiones: valoración de propias emociones, núcleo del burnout y falta de realización. En este sentido, a menor núcleo del burnout, mayor valoración de propias emociones. De igual manera, a menor falta de realización, mayor valoración de propias emociones.

Tabla 1

Matriz de correlaciones no paramétricas de las variables inteligencia emocional y burnout

\begin{tabular}{llllllllllll}
\hline Dimensiones & 1 & 2 & 3 & 4 & 5 & 6 & 7 & 8 \\
\hline
\end{tabular}

1. Núcleo del burnout

2. Falta de realización $.52^{* *}-$ 

3. Uso de las propias emociones $-.38^{* *}-.33^{* *}-$
4. Regulación de emociones $-.28^{* *}-.26^{* *} \quad .57^{* *}-$

5. Valoración de propias emociones $-.25^{* *} \quad-.21^{* *} \quad .56^{* *} \quad .57^{* *}-$

6. Valoración de las emociones de otros

7. Edad

8. Tiempo de Servicio $* * \mathrm{p}<.01$ $* \mathrm{p}<.05$

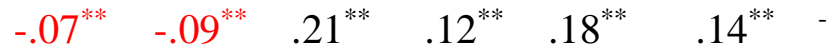

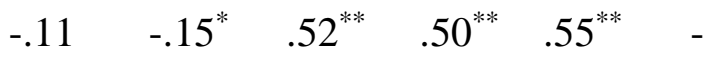

$-.13^{* *}-.14^{* *} \quad .23^{* *} \quad .12^{* *} \quad .23^{* *} \quad .16^{* *} \quad .87^{* *} \quad-$

Por otro lado, en la misma tabla 1 se observa las relaciones que existen entre las dimensiones y las variables sociodemográficas de edad y tiempo de servicio. Respecto a la variable edad, se percibe la relación que existe con la dimensión uso de las propias emociones de inteligencia emocional. Tomando en cuenta la variable tiempo de servicio, se identifica una correlación negativa y de baja magnitud en relación a las dimensiones del burnout. Cabe señalar que existe una mayor relación con la inteligencia emocional; específicamente con las dimensiones uso de las propias emociones y valoración de propias emociones.

En la tabla 2, se evidencia las comparaciones de las dimensiones de inteligencia emocional, burnout y la variable situación laboral. Así, se identifican diferencias entre la falta de realización personal en función a la situación laboral. Sin embargo, no existieron diferencias significativas respecto al núcleo del burnout. Asimismo, se observa que existen diferencias en la valoración de las propias emociones de acuerdo a la situación laboral. El cálculo del tamaño del efecto indicó una ausencia del mismo.

Tabla 2

Diferencias en las variables inteligencia emocional y burnout según situación laboral

\begin{tabular}{lllllll}
\hline \multirow{2}{*}{ Dimensión } & \multicolumn{2}{c}{ Situación Laboral } & & & & \\
& & & U & P & RP \\
\cline { 2 - 5 } & Nombrada Contratada & & & & \\
\hline
\end{tabular}


Núcleo del

burnout

139.97

151.74

9166

$-1.14 \quad .25$

.46

Falta de realización

132.07

156.20

8328

$-2.35 \quad .02$

.42

Uso de las

propias

emociones

162.09

139.27

8417

$-2.23 \quad .03$

.42

Regulación de emociones

155.18

143.17

9150

$-1.17 \quad .24$

.46

Valoración de propias emociones

162.91

138.81

$\begin{array}{lll}8330.50 & -2.35 & .02\end{array}$

.42

Valoración de las emociones de los otros $159.45 \quad 140.76$ $\begin{array}{lll}8697.50 & -1.82 \quad .07\end{array}$

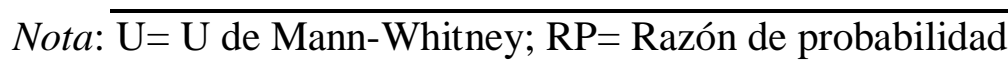

Respecto a la comparación de las dimensiones de inteligencia emocional, burnout y la variable estudios de segunda especialidad y diplomados, la tabla 3 indica la existencia de diferencias respecto al uso de las propias emociones. No obstante, no presentan también tamaño del efecto importante.

Tabla 3

Diferencias en las variables inteligencia emocional y burnout según estudios de segunda especialidad y diplomados

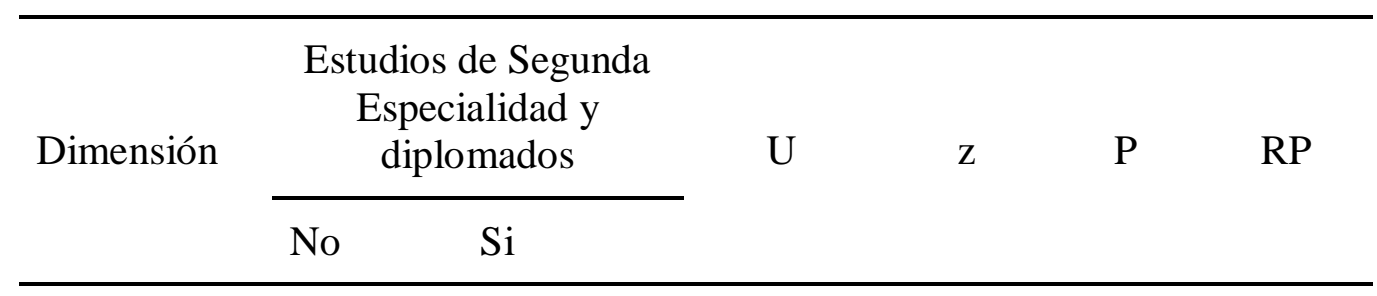




\begin{tabular}{|c|c|c|c|c|c|c|}
\hline $\begin{array}{l}\text { Núcleo del } \\
\text { burnout }\end{array}$ & 155.03 & 142.73 & 9401.50 & -1.21 & .23 & .46 \\
\hline $\begin{array}{l}\text { Falta de } \\
\text { realización }\end{array}$ & 153.29 & 143.84 & 9600.50 & -.93 & .35 & .47 \\
\hline $\begin{array}{l}\text { Uso de las } \\
\text { propias } \\
\text { emociones }\end{array}$ & 133.07 & 156.64 & 8615 & -2.33 & .02 & .42 \\
\hline $\begin{array}{l}\text { Regulación } \\
\text { de } \\
\text { emociones }\end{array}$ & 140.01 & 152.24 & 9406.50 & -1.21 & .23 & .46 \\
\hline $\begin{array}{l}\text { Valoración } \\
\text { de propias } \\
\text { emociones }\end{array}$ & 138.82 & 153 & 9270.50 & -1.40 & .16 & .45 \\
\hline $\begin{array}{l}\text { Valoración } \\
\text { de las } \\
\text { emociones } \\
\text { de los otros }\end{array}$ & 142.91 & 150.41 & 9737 & -.74 & .46 & .47 \\
\hline
\end{tabular}

Nota: $\mathrm{U}=\mathrm{U}$ de Mann-Whitney; RP= Razón de probabilidad

En la tabla 4, se registran las diferencias existentes entre las dimensiones de inteligencia emocional, burnout y la variable apoyo de un auxiliar en el aula. En primer lugar, se identificó las diferencias entre la variable y la dimensión falta de realización. Luego, se evidenció las diferencias entre la variable y la dimensión uso y valoración de las propias emociones. Sin embargo, no se presenta tamaño del efecto.

Tabla 4

Diferencias en las variables inteligencia emocional y burnout según el apoyo de un auxiliar en el aula

\begin{tabular}{llllll}
\hline Dimensión & Auxiliar & $\mathrm{U}$ & $\mathrm{z}$ & $\mathrm{p}$ & $\mathrm{RP}$ \\
\hline
\end{tabular}




\begin{tabular}{|c|c|c|c|c|c|c|}
\hline & $\mathrm{Si}$ & No & & & & \\
\hline $\begin{array}{l}\text { Núcleo del } \\
\text { burnout }\end{array}$ & 140.38 & 158.58 & 9018.50 & -1.79 & .07 & .44 \\
\hline $\begin{array}{l}\text { Falta de } \\
\text { realización }\end{array}$ & 138.53 & 161.47 & 8686 & -2.27 & .02 & .42 \\
\hline $\begin{array}{l}\text { Uso de las } \\
\text { propias } \\
\text { emociones }\end{array}$ & 160.63 & 127.06 & 7942 & -3.33 & .00 & .39 \\
\hline $\begin{array}{l}\text { Regulación } \\
\text { de } \\
\text { emociones }\end{array}$ & 151.27 & 141.63 & 9617.50 & -.95 & .34 & .47 \\
\hline $\begin{array}{l}\text { Valoración } \\
\text { de propias } \\
\text { emociones }\end{array}$ & 155.44 & 135.13 & 8870.50 & -2.01 & .04 & .43 \\
\hline $\begin{array}{l}\text { Valoración } \\
\text { de las } \\
\text { emociones } \\
\text { de los otros }\end{array}$ & 154.59 & 136.46 & 9023 & -1.79 & .07 & .44 \\
\hline
\end{tabular}

Nota: $\mathrm{U}=\mathrm{U}$ de Mann-Whitney; RP= Razón de probabilidad

\section{Discusión}

Los hallazgos indican que existe relación negativa entre las dimensiones uso, regulación y valoración de las propias emociones de la inteligencia emocional y las dimensiones núcleo del burnout y falta de realización del burnout. Es decir, las docentes que poseen mayor grado de inteligencia emocional podrán gestionar de mejor manera las demandas laborales y, a su vez, la posibilidad de relacionarse con los distintos agentes de los centros educativos de manera apropiada; esto permitirá afrontar adecuadamente el agotamiento laboral. Asimismo, accederá a las docentes sentirse realizadas al momento de desempeñarse en sus centros laborales. Esto se asemeja a los hallazgos encontrados en el estudio de Serrano et al. (2018) quiénes reportan la presencia de una correlación negativa y estadísticamente significativa entre las competencias emocionales y el burnout en una muestra de docentes de educación inicial de España. Por su parte, Aguado (2016) indica que los docentes con altos niveles de inteligencia emocional poseen un 
adecuado bienestar laboral y personal, lo cual les permite potenciar sus competencias personales, rasgos de personalidad, seguridad en sí mismas, habilidades sociales y otros aspectos vinculados al rol principal que ejercen en la educación; sea como agentes de enseñanza y aprendizaje que promuevan y brinden un ambiente educativo de calidad (Ruiz, 2016).

En otro orden de cosas, se observó una ausencia de correlación entre la dimensión valoración de emociones en otros, perteneciente a inteligencia emocional, y burnout. En este sentido, la identificación y el reconocimiento de las emociones de los demás, no influye en el modo en que la docente maneja su desgaste laboral, pues son aspectos que se ubican fuera del alcance de cada una de ellas. Dicho resultado corrobora lo hallado por Torres y Cobo (2016), quienes sostienen que, si las maestras son capaces de tener autoconciencia, emociones positivas y adecuada gestión de la automotivación, podrán tener un desempeño eficaz en su entorno y afrontar el burnout. Este último aspecto se enfoca en las oportunidades individuales de las docentes, excluyendo las acciones o pensamientos que involucran a otros. Cabe señalar que los resultados hallados no concuerdan con lo mencionado por Herrero (2017), quien detalla que una de las características de inteligencia emocional es la empatía, poniendo énfasis en la importancia de reconocer y comprender al otro.

Es necesario incidir que, en este estudio y en el de Serrano et al. (2018), se considera únicamente a dos dimensiones del burnout debido al alfa de cronbach de una de ellas, ya que obtuvo un valor menor a lo aceptado. Asimismo, en otros estudios sobre el burnout relacionado con otras variables al momento de analizar la estructura interna del instrumento, terminan considerando a dos dimensiones o en otras a una sola (Gil, 2018; Zuazo, 2018). Es importante mencionar la importancia de la confiabilidad de una prueba psicológica, la cual permite precisar el nivel de evidencia y precisión vinculada a las puntuaciones del instrumento y analizar posibles errores de medición (Ventura-León, 2017).

Otros resultados afirman que existe una relación entre la edad y el uso de las propias emociones; no obstante, ésta no guarda relación con burnout. Es necesario mencionar que estos resultados no concuerdan con lo detallado por De Vera García y Gabari (2019), quienes explican que la edad guarda relación con burnout, específicamente en aquellos docentes que se insertan por primera vez en el campo laboral, debido a que no cuentan con experiencias previas, generándoles ansiedad y tensión al enfrentarse a este nuevo contexto. De igual manera, se identifica una relación entre el tiempo de servicio, el uso de las propias emociones y valoración de las mismas, pero no con el burnout. Sin embargo, Suárez y Martín (2019), señalan que las docentes con mayores años 
de experiencia pueden llegar a manifestar actitudes vinculadas al burnout; tales como apatía y rechazo hacia el propio desempeño.

Por otro lado, se identificaron diferencias significativas en la falta de realización según la situación laboral, resultado que no se observó en la dimensión núcleo del burnout. Asimismo, se evidencian diferencias en el uso de las propias emociones y valoración de propias emociones de acuerdo a la situación laboral. De Vera García y Gabari (2019), explican que la situación laboral de las docentes contratadas influye negativamente en su desempeño, ya que presentan mayor agotamiento y estrés al no contar con un cargo permanente.

Si bien se reportó que el uso de las propias emociones varía en función a los estudios de segunda especialidad y diplomados, no existen investigaciones previas que sustenten dicho resultado. Finalmente, las docentes que no cuentan con el apoyo de una auxiliar presentan una mayor falta de realización; en cambio, aquellas que poseen dicha ayuda poseen mayores capacidades al momento de manejar y comprender sus emociones. Es necesario señalar estudios que determinan diferencias entre los niveles de burnout de las docentes y auxiliares; no obstante, no existe evidencia en relación a este estudio (Choy, 2017).

En otro orden, es importante considerar algunas limitaciones. Primero, son escasas las investigaciones que contengan características similares a la muestra de la presente investigación. Segundo, existieron ciertas restricciones para acceder a cada institución educativa debido a que algunas solicitaban documentos de presentación; asimismo, algunos colegios se encontraban lejanos al centro de la ciudad. Tercero, debido al uso de autoinformes existe una mayor probabilidad de sesgos por deseabilidad social, en lo cual el sujeto encuestado brinda una respuesta en función de una posible aceptabilidad, sin poner en práctica un criterio y/o reflexión (Campos \& Rueda, 2017). Cuarto, solo se evaluó a docentes de inicial de un departamento de la sierra sur del Perú, por lo que los resultados no pueden generalizarse a todas las docentes del nivel inicial del país. A pesar de las limitaciones, los resultados servirán como base para la elaboración de evaluaciones y programas de intervención que permitan promocionar las habilidades emocionales de las docentes, a fin de disminuir las consecuencias del burnout y mejorar la relación docentealumno.

Se concluye que las dimensiones de inteligencia emocional y burnout se relacionan de manera negativa excluyendo la dimensión valoración de emociones en otros. Respecto a los objetivos específicos, se encuentra correlaciones positivas entre edad y uso de sus propias 
emociones perteneciente a la inteligencia emocional, del mismo se evidencia que a mayor tiempo de servicio, mayor uso y valoración de las propias emociones. Por otro parte, al comparar la inteligencia emocional y el burnout con la situación laboral, se confirmó que las docentes nombradas se sienten realizadas, usan y valoran sus emociones, a diferencia de las contratadas. En relación a los estudios de segunda especialidad y diplomados, las docentes que poseen dicha formación usan sus propias emociones, a diferencia de aquellas que no cuentan con dichos estudios. Finalmente, las maestras con auxiliar en el aula usan y valoran sus emociones, y se sienten realizadas; contrariamente a las que no cuentan con auxiliar.

Por tanto, se recomienda que futuros estudios evalúen a muestras de docentes de otras regiones del Perú con el propósito de corroborar los datos hallados, debido a las características de la población seleccionada y el acceso restringido que se tiene a ella. De igual manera, se debería ejecutar programas de intervención que continúen fortaleciendo la inteligencia emocional de las docentes y técnicas del manejo de estrés para evitar la aparición del burnout.

\section{Referencias}

Aguado, J. (2016). Inteligencia emocional en docentes de instituciones educativas de la ciudad de Pampas. (Tesis de maestría). Universidad Nacional del Centro del Perú, Huancayo.

Almeida, M., Nazaré, E., Rosemiro, F., Evangelista, R., Gomes, A., \& Vieira, B. (2015). Síndrome de burnout: un estudio con profesores. Salud de los Trabajadores, 23(1), 19-27.

Arias, W., Huamani, J., \& Ceballos, K. (2019). Síndrome de Burnout en profesores de escuela y universidad: un análisis psicométrico y comparativo en la ciudad de Arequipa. Propósitos y Representaciones, 7(3), 72-91. doi: 10.20511/pyr2019.v7n3.390

Barcelar, L., \& Martín, M. (2019). Formación inicial docente y competencias emocionales. Educação e Pesquisa 45. doi: 10.1590/s1678-4634201945186508

Campos, M., \& Rueda, F. (2017). Sesgo de deseabilidad social en medidas de valores organizacionales. Universitas Psychologica, 16(2), 1-11. doi: 10.11144/Javeriana.upsy162.sdsm

Choy, R. (2017). Burnout y desempeño laboral en docentes universitarios de una carrera en una universidad privada de Lima Metropolitana. (Tesis de maestría). Universidad Peruana Cayetano Heredia, Lima.

Davies, M., Stankov, L., \& Roberts R. (1998). Emotional intelligence: in search of an elusive construct. Journal of Personality and Social Psychology, 75(4), 989-1015. doi: 10.1037/0022-3514.75.4.989 
De Vera García, I., \& Gabari, I. (2019). Niveles de Burnout en docentes de secundaria: un estudio descriptivo analítico. Revista INFAD de Psicología, 2(1), 455-464.

Faul, F., Erdfelder, E., Lang, A.-G., \& Buchner, A. (2007). G*Power 3: A flexible statistical power analysis program for the social, behavioral, and biomedical sciences. Behavior Research Methods, 39(2), 175-191. doi: 10.3758/BF03193146

García, A., Escorcia, C., \& Pérez, B. (2017). Síndrome de burnout y sentimiento de autoeficacia en profesores universitarios. Propósitos y Representaciones, 5(2), 65-126. doi: 10.20511/pyr2017.v5n2.170

Gil, L. (2018). Síndrome de burnout y autoeficacia en tripulantes de cabina de Lima. (Tesis de licenciatura). Universidad Peruana de Ciencias Aplicadas, Lima.

Grissom, R. (1994). Probability of the superior outcome of one treatment over another. Journal of Applied Psychology, 79(2), 314-316. doi: 10.1037/0021-9010.79.2.314

Guadalupe, C., León, J., Rodríguez, J., \& Vargas, S. (2017). Estado de la Educación en el Perú: Análisis y Perspectivas de la Educación Básica. Lima, Perú: Grade.

Herrero, C. (2017). Habilidades sociales y nivel de burnout en profesionales de enfermería de triaje del Hospital Universitario Central de Asturias. (Tesis de maestría). Universidad de Oviedo, España.

Ilaja, B., \& Reyes, C. (2016). Burnout y estrategias de inteligencia emocional en profesores universitarios: implicados en la salud laboral educativa. Psicología desde el Caribe, 33(1), 31-46.

Markova, E. (3 de febrero de 2017). La educación, una prioridad de desarrollo a nivel mundial, regional y nacional [Entrada en Blog]. Recuperado de

https://blogs.worldbank.org/es/opendata/la-educacion-una-prioridad-de-desarrollo-a-nivelmundial-regional-y-nacional [Consulta: 12 de setiembre de 2019].

Maslach, C., \& Jackson, S. (1981). The measurement of experience burnout. Journal of Occupational Behavior, 2(2), 99-103. doi: 10.1002/job.4030020205

Mayer, J., \& Salovey, P. (1997). Emotional development and emotional intelligence: educational implications. New York, NY: Basic books.

Merino, C., Lunahuaná-Rosales, M., \& Kumar, R. (2016). Validación estructural del Wong-Law emotional intelligence scale (WLEIS): Estudio preliminar en adultos. Liberabit, 22(1), 103-110.

Ministerio de Educación (MINEDU). (2015). Ayacucho: ¿Cómo vamos en educación?. Unidad de Estadística, MINEDU. Recuperado de http://repositorio.minedu.gob.pe/bitstream/handle/123456789/4701/Ayacucho\%20c\%C3\% B3mo\%20vamos\%20en\%20educaci\%C3\%B3n.pdf?sequence=1\&isAllowed=y

Ministerio de Educación (MINEDU). (2016). Currículo nacional de la educación básica. Lima, Perú: (C) Ministerio de Educación. 
Ministerio de Educación (MINEDU). (2017). Presentación del proceso censal 2017 - Ayacucho. Estadística de la Calidad Educativa (ESCALA). Recuperado de http://escale.minedu.gob.pe/c/document_library/get_file?uuid=1a32fd96-ddf9-4efb-891e2f14a133cd1f\&groupId=10156

Moreno-Jiménez, B., Garrosa, E., \& González, J. (2000). La evaluación del estrés y el burnout del profesorado: el CBP-R. Revista de psicología del trabajo y las organizaciones, 16(2), 151-171.

Moreno-Jiménez, B., Corzo, S., Sanz-Vergel, A., Rodríguez-Muñoz, A., \& Boada, M. (2010). El "Burnout" y el "Engagement" en profesores de Perú. Aplicación del modelo de demandas recursos laborales. Ansiedad y Estrés, 16(2-3), 293-307.

Otzen, T., \& Manterola, C. (2017). Técnicas de muestreo sobre una población a estudio. International Journal of Morphology, 35(1), 227 - 232. doi: 10.4067/S071795022017000100037

Posada, J., Molano, P., Parra, R., Brito, F., \& Rubio, E. (2019). Prevalencia del Síndrome de Burnout en docentes: Factores asociados al estatuto de vinculación laboral en Colombia. Revista Interamericana de Psicología Ocupacional, 37(2), 119-133. doi: 10.21772/ripo.v37n2a04

Ruiz, J. (2016). El bienestar emocional del docente. Revista nacional e internacional de educación inclusiva, 9(2), 183-194.

Serrano, N., Pocinho, M., \& Aragón, E. (2018). Competencias emocionales y síndrome de burnout en el profesorado de educación infantil. Revista de Psicología y Educación, 13(1), 1-12. doi: 10.23923/rpye2018.01.153

Suárez, M., \& Martín, J. (2019). Influencia del perfil sociodemográfico del profesorado universitario sobre la inteligencia emocional y el burnout. Educación XX1, 22(2), 93-117. doi: 10.5944/educXX1.22514

Torres, P., \& Cobo, J. (2016). Estrategias de gestión de la inteligencia emocional para la prevención del Síndrome de Burnout en docentes de aula. Educ@ción en contexto, 2(especial), 281-295.

Usán, P., \& Salavera, C. (2019). El rendimiento escolar, la inteligencia emocional y el engagement académico en adolescentes. Revista electrónica de Investigación Psicoeducativa, 17(1), 5-26.

Ventura-León, J. (2017). La importancia de reportar la validez y confiabilidad en los instrumentos de medición: Comentarios a Arancibia et al. Revista médica de Chile, 145(7), 955-956. doi: 10.4067/s0034-98872017000700955

Wong, C., \& Law, K. (2002). The effects of leader and follower emotional intelligence on performance and attitude: An exploratory study. Leadership Quarterly, 13(3), 243-274.

Zuazo, A. (2018). Burnout y calidad de vida profesional en policías de Lima Metropolitana. (Tesis de licenciatura). Universidad Peruana de Ciencias Aplicadas, Lima. 